\title{
Fractional Superstrings with Space-Time Critical Dimensions Four and Six
}

\author{
Philip C. Argyres and S.-H. Henry Tye \\ Newman Laboratory of Nuclear Studies, Cornell University, Ithaca, New York 14853-5001 \\ (Received 8 August 1991; revised manuscript received 11 September 1991)
}

\begin{abstract}
We propose possible new string theories based on local world-sheet symmetries corresponding to extensions of the Virasoro algebra by fractional-spin currents. They have critical central charges $c=6(K+8) /(K+2)$ and Minkowski space-time dimensions $D=2+16 / K$ for $K \geq 2$ an integer. We present evidence for their existence by constructing modular-invariant partition functions and the massless particle spectra. The dimension 4 and 6 strings have space-time supersymmetry.
\end{abstract}

PACS numbers: 11.17.+y

String theories [1] are primarily characterized by the local symmetries of a two-dimensional field theory on the string world sheet. The local symmetries of the bosonic string are reparametrization and Weyl invariance which lead to a critical string propagating in 26 flat space-time dimensions. The superstring enlarges the world-sheet gauge invariance to include a local $N=1$ supersymmetry, and reduces the critical space-time dimension to 10 . It is natural to try to construct string theories with smaller critical space-time dimensions by changing the worldsheet symmetry. Unfortunately, enlarging to a local $N=2$ supersymmetry produces a critical string in just two-dimensional Minkowski space. It is well known, however, that fractional-spin fields can exist in twodimensional field theory. One can imagine new local symmetries on the world sheet which involve fractionalspin currents and which lead to string propagation in space times with dimensions less than 10. In this Letter we will present evidence for the existence of such new string theories, and, in particular, will show that strings with spin- $\frac{4}{3}$ and $-\frac{6}{5}$ currents on the world sheet can have interesting phenomenologies in 6 and 4 space-time dimensions, respectively.

The algebra of constraints resulting from gauge fixing the two-dimensional local symmetry must contain the Virasoro algebra. In this Letter we consider strings with the Virasoro algebra extended by chiral currents with fractional spins (equivalently, conformal dimensions) given by $\Delta=1+2 /(K+2)$ for $K \geq 2$ an integer. These fractional-spin currents transform bosonic fields to spin$2 /(K+2)$ fields. We refer to these algebras as fractional superconformal algebras and the string theories based on these algebras as fractional superstrings. The $K=2$ case is the usual superstring with the super Virasoro algebra as its constraint algebra. The construction of fractional superstrings generalizes that of the superstring.

In fact, the fractional superconformal algebras were proposed a few years ago $[2,3]$; we can construct them as follows. Consider the chiral $\mathrm{SU}(2)_{K}$ Wess-ZuminoWitten (WZW) theory. Denote the WZW primary fields of spin $j$ and $J_{3}$ quantum number $m$ by $\Phi_{m}^{j}$, for $0 \leq j \leq K / 2$, and the $n$th mode of the Kac-Moody currents by $J_{n}^{m}$. Define the current [2] $\hat{G}^{K}(z)=\sum_{m} J_{-1}^{m}$ $\times \Phi_{m}^{l}(z)$. The WZW theory can be expressed as the ten- sor product of a free scalar field $\phi$, compactified on a circle of radius $1 / \sqrt{K}$, and the $Z_{K}$-parafermion theory [4]. The parafermion fields can be organized into sets $f_{m}^{j}$ according to $\mathrm{SU}(2)_{K}$ quantum numbers. In terms of these fields $\hat{G}_{K}$ can be expressed as

$$
\hat{G}^{K}(z)=\epsilon(z) \partial \phi(z)+\eta(z),
$$

where $\epsilon$, the lowest dimension member of $f_{0}^{1}$, is the first energy operator of the $Z_{K}$-parafermion theory, and has dimension $2 /(K+2)$. The $\eta$ field (present for $K \geq 3$ ) is a parafermion descendant of $\epsilon$, but Virasoro primary with dimension $\Delta$.

The chiral algebra generated by the energy-momentum tensor and $\hat{G}_{K}$ (appropriately modified with background charge for $\phi$ ) was first proposed as the underlying symmetry of the $\mathrm{SU}(2)_{K} \otimes \mathrm{SU}(2)_{L} / \mathrm{SU}(2)_{K+L}$ coset models $[2,3]$. Much evidence has accumulated showing the importance of these algebras in organizing the operator content of conformal field theories [5-7]. We should emphasize, however, that except in some special cases $[5,8]$ the exact form of this fractional supersymmetry algebra is not known.

Next, we replace the scalar field $\phi$ with a decompactified one called $X$. The SU(2) WZW symmetry is now lost. We interpret the $X$ field as the string coordinate and tensor together $D$ copies to allow a $D$-dimensional space-time interpretation. Adding space-time indices, we write the (world-sheet) fractional supersymmetry current for a $D$-dimensional fractional superstring as

$$
G^{K}(z)=\epsilon^{\mu} \partial X_{\mu}(z)+: \epsilon^{\mu} \epsilon_{\mu}(z):
$$

where the index $\mu=0,1,2, \ldots, D-1$ is contracted with a Minkowski metric. The normal ordering symbol in the second term is meant as an instruction to pick out the $\eta$ field which appears as the dimension $\Delta$ operator in the $\epsilon \epsilon$ operator product expansion [see also Eq. (4) below]. Thus, the conformal field theory corresponding to a single space-time dimension is a $c=1$ free boson plus a $c=2(K-1) /(K+2) Z_{K}$-parafermion theory and has a conformal anomaly $c_{0}=3 K /(K+2)$. It is easy to show that the $D$-fold tensor product algebra generated by $G^{K}$ closes on itself if the single component algebra $\hat{G}_{K}$ does [9]. 
For $K=2$, the $Z_{K}$-parafermion theory is just the Ising model and the energy operator $\epsilon$ is simply the free Majorana-Weyl field; the second term in Eq. (2) vanishes in this case. This describes the usual superstring.

We now wish to show that in addition to the $K=2$ superstring, theories with other $K$ values can have sensible interpretations as string theories in flat space-time. To this end, we follow a simple argument to determine the space-time critical dimension of the fractional superstring as a necessary condition for a desirable string phenomenology. Along the way we show that the fractional supercurrent imposes the correct physical state condition on the massless particles in the spectrum.

The physical state conditions arising from the gaugefixing constraints on the world sheet are $G_{n}^{K} \mid$ phys $\rangle$ $=L_{n} \mid$ phys $\rangle=0$ for $n>0$ and $L_{0} \mid$ phys $\rangle=v \mid$ phys $\rangle$, where the $L_{n}$ are the generators of the Virasoro algebra and $v$ is the intercept. To determine $v$, we demand that the open string theory have a massless vector particle (a graviton in the closed string theory). For example, in the bosonic string, the lowest-mass vector particle is $|\psi\rangle=\alpha_{-}^{\mu}|p\rangle$, where $\alpha_{n}^{\mu}$ are the modes of the $X^{\mu}$ coordinate fields and $|p\rangle$ is the ground state with momentum $p^{v}$. To be a physical state, it must satisfy $0=\left(L_{0}-v\right)|\psi\rangle=\left(1-M^{2}\right.$ $-v)|\psi\rangle$. Therefore $|\psi\rangle$ is massless only for $v=1$. Now apply this argument to the fractional superstring. For $K \geq 2$ the lowest-mass vector particle is

$$
|\psi\rangle=\zeta_{\mu} \epsilon_{-2 /(K+2)}^{\mu}|p\rangle,
$$

where the moding of the energy operator $\epsilon^{\mu}$ acting on the ground state follows from its dimension and $\zeta_{\mu}$ is the polarization tensor. The physical state condition $0=\left(L_{0}\right.$ $-v)|\psi\rangle=\left[2 /(K+2)-M^{2}-v\right]|\psi\rangle$ implies that $|\psi\rangle$ is massless when $v=2 /(K+2)$.

The only nontrivial physical state condition on $|\psi\rangle$ is $0=G_{2 /(K+2)}^{K}|\psi\rangle$. This can be computed using the mode expansion of the fractional supersymmetry current following from Eq. (2),

$$
G_{2 /(K+2)}^{K}=\sum_{n} \epsilon_{2 /(K+2)+n}^{\mu}\left(\alpha_{-n, \mu}+c_{n} \epsilon_{-n, \mu}\right),
$$

where the $\alpha_{n}$ are the $X$-boson modes and the $c_{n}$ are coefficients that can be calculated from the $Z_{K}$-parafermion theory. Since the second term annihilates $|\psi\rangle$, we find for the physical state condition $0=G_{2 /(K+2)}^{K}|\psi\rangle$ $=p \cdot \zeta|p\rangle$. Because $|\psi\rangle$ is massless, the longitudinal state with $\zeta^{\mu} \propto p^{\mu}$ is null. Thus we find the physical state condition and the $D-2$ propagating degrees of freedom appropriate for a massless vector particle.

To be consistent with the massless vector particle, we require that, for the whole spectrum, effectively only $D-2$ transverse dimensions worth of polarization states actually propagate. This is a signal of the enlarged gauge invariance of critical strings.

The character $\chi(q)$ which includes the string ground state counts the number of propagating degrees of freedom $N$ at each mass level $M^{2}$ by the coefficients of terms in a power series expansion in $q$ :

$$
\chi(q)=\sum N q^{M^{2}}=q^{-v}(1+\cdots) .
$$

The second equality follows from the fact that $L_{0}$ $=-M^{2}+\cdots$ counts the mass level of a state, where the dots stand for Fock space number operators. Thus the lowest-mass physical state obeys $M^{2}=-v$. On the other hand, conformal invariance requires that [10]

$$
\chi(q)=q^{-c_{\mathrm{eff}} / 24}(1+\cdots),
$$

where $c_{\text {eff }}$ is the effective conformal anomaly of the propagating degrees of freedom. From the requirement that only the transverse dimensions couple in a critical string, we have $c_{\text {eff }}=(D-2) c_{0}$. Comparison of Eqs. (5) and (6) shows that $D=2+24 v / c_{0}$.

Since $c_{0}=3 K /(K+2)$ for the fractional superstring, and we found $v=2 /(K+2)$ above, we have for $K \geq 2$

and

$$
D=2+16 / K
$$

$$
c_{\text {crit }}=D c_{0}=6(K+8) /(K+2) .
$$

For $K=2,4,8,16$, and $\infty$, we find the new integer critical dimensions $D=10,6,4,3$, and 2, respectively [11]. Curiously, these are precisely the dimensions which allow minimal super Yang-Mills theories. Note that for $K=2$ we recover the superstring result $D=10$.

We will now construct modular-invariant partition functions for the $K=4$ and 8 closed fractional superstrings. As above, we impose the conditions that only $D-2$ dimensions worth of states propagate, and that there is a graviton in the closed string spectrum. In addition, we require that no tachyonic states appear in the spectrum.

Each of the $D-2$ transverse dimensions contribute a $Z_{K}$-parafermion plus $X^{\mu}$-boson worth of states to the partition function. Specifically, each boson contributes a factor $\eta(q)^{-1}$, the reciprocal of the Dedekind eta function, and each set of parafermion fields $f_{m}^{j}$ contributes a factor $\eta(q) c_{2 m}^{2 j}(q)$ to the partition function, where the $c_{2 m}^{2 j}$ are string functions [12]. The string functions obey the identities $c_{K}^{K-2 j}=c_{2 m}^{2 j}=c^{2 j} 2 m$, and have known power series expansions in $q$ starting with $c_{2 m}^{2 j}(q)=q^{h(j, m)}(1$ $+\cdots)$ where, for $|m| \leq j$,

$$
h(j, m)=\frac{8 j(j+1)-K}{8(K+2)}-\frac{m^{2}}{K} .
$$

The partition function must also be invariant under the modular transformations $T: \tau \rightarrow \tau+1$ and $S: \tau$ $\rightarrow-1 / \tau$, where $q=\exp (2 i \pi \tau)$. The $T$ modular transformation properties of the string functions follow from Eq. (9), while the $S$ transformation is given by [12]

$$
c_{2 m}^{2 j}(-1 / \tau)=[-i \tau K(K+2)]^{-1 / 2} \sum_{J=0}^{K} \sum_{M=1-K}^{K} e^{2 i \pi m M / K} \sin \left[\frac{\pi(2 j+1)(J+1)}{K+2}\right] c_{M}^{J}(\tau) \text {. }
$$


Let us briefly review the construction of the partition function of the $K=2$ (superstring) theory with critical dimension $D=10$. The partition function will be a sum of terms each with eight $Z_{2}$-parafermion (free-fermion) string function factors for each of the eight transverse dimensions. A tachyon-free modular-invariant partition function is found [13] to be $Z_{(2,2)}(q)=\left|A_{2}\right|^{2}$, where [14]

$$
\begin{aligned}
A_{2}= & 8\left(c_{0}^{0}\right)^{7} c_{0}^{2}-8\left(c_{1}^{1}\right)^{8}+56\left(c_{0}^{0}\right)^{5}\left(c_{0}^{2}\right)^{3} \\
& +56\left(c_{0}^{0}\right)^{3}\left(c_{0}^{2}\right)^{5}+8 c_{0}^{0}\left(c_{0}^{2}\right)^{7} .
\end{aligned}
$$

From Eq. (9) we see that only the first two terms contribute to the massless spectrum, and the requirement that the left-moving spectrum contain a massless vector particle (or, equivalently, that $Z_{(2,2)}$ contain the graviton) fixes the normalization of $A_{2}$. The translation to the Jacobi $\theta$-function notation is $\eta^{3}\left(c_{0}^{0}+c_{0}^{2}\right)^{2}=\theta_{3}$ and $\eta^{3}\left(c_{0}^{0}\right.$ $\left.-c_{0}^{2}\right)^{2}=\theta_{4}$ for the Neveu-Schwarz sector and $2 \eta^{3}\left(c_{1}^{1}\right)^{2}$ $=\theta_{2}$ for the Ramond sector. The massless sector in $Z_{(2,2)}$ contains an $N=2$ supergravity multiplet and $A_{2}$ vanishes, consistent with space-time supersymmetry. We will find that all of these features are present in the $K=4$ and $K=8$ partition functions.

The $K=4$ fractional superstring has critical dimension $D=6$, conformal anomaly $c_{0}=2$ per space-time dimension, and a fractional supercurrent $G^{K}$ of dimension $\frac{4}{3}$. We find one tachyon-free modular-invariant partition function

$$
Z_{(4,4)}(q)=\left|A_{4}\right|^{2}+12\left|B_{4}\right|^{2},
$$

with

$$
\begin{aligned}
A_{4}= & 4\left(c_{0}^{0}+c_{0}^{4}\right)^{3} c_{0}^{2}-4\left(c_{2}^{2}\right)^{4}+32\left(c_{2}^{0}\right)^{3} c_{2}^{2}-4\left(c_{0}^{2}\right)^{4}, \\
B_{4}= & -4\left(c_{2}^{2}\right)^{2}\left(c_{0}^{2}\right)^{2}+8\left(c_{0}^{0}+c_{0}^{4}\right) c_{0}^{2}\left(c_{2}^{0}\right)^{2} \\
& +4\left(c_{0}^{0}+c_{0}^{4}\right)^{2} c_{2}^{2} c_{2}^{0} .
\end{aligned}
$$

Under the $S$ modular transformation the partition function blocks obey $S\left(A_{4}\right)=\frac{1}{2} A_{4}+3 B_{4}$ and $S\left(B_{4}\right)=\frac{1}{4} A_{4}$ $-\frac{1}{2} B_{4}$. Equation (9) implies that $A_{4} \sim q^{0}(1+\cdots)$ and $B_{4} \sim q^{1 / 2}(1+\cdots)$. Thus we see that there are no tachyons in this theory, and that the only contributions to the massless states are from the terms $4\left(c_{0}^{0}\right)^{3} c_{0}^{2}-4\left(c_{2}^{2}\right)^{4}$ in $A_{4}$. The first term has the interpretation as the massless vector particle since it is created from the $\left(c_{0}^{0}\right)^{6}$ vacuum by a parafermion field with $j=1$ and $m=0$, giving rise to one $c_{0}^{2}$ factor. These are precisely the quantum numbers of the $\epsilon^{\mu}$ energy operators, so we can identify it with the massless vector state of Eq. (3). The number of degrees of freedom of a massless vector particle in six dimensions is four, fixing the normalization of the partition function. The second term, appearing with a minus sign, must be interpreted as a space-time fermion. It is composed of $j=1$ spin fields in the parafermion theory, commonly denoted $\sigma_{2} \in f_{1}^{1}$. The normalization of this term suggests that it is a space-time spin- $\frac{1}{2}$ Weyl field.

Entirely similar observations hold for the $K=8$ frac- tional superstring. This string has critical dimension $D=4$, conformal anomaly $c_{0}=\frac{12}{5}$ per space-time dimension, and a fractional supercurrent $G^{K}$ of dimension $\frac{6}{5}$. For the $K=8$ closed fractional superstring, we find one tachyon-free modular-invariant partition function

$$
Z_{(8,8)}(q)=\left|A_{8}\right|^{2}+\left|B_{8}\right|^{2}+2\left|C_{8}\right|^{2},
$$

where

$$
\begin{aligned}
& A_{8}=2\left(c_{0}^{0}+c_{0}^{8}\right)\left(c_{0}^{2}+c_{0}^{6}\right)-2\left(c_{4}^{4}\right)^{2}+8 c_{4}^{0} c_{4}^{2}-2\left(c_{0}^{4}\right)^{2}, \\
& B_{8}=4\left(c_{0}^{0}+c_{0}^{8}\right) c_{4}^{2}+4\left(c_{0}^{2}+c_{0}^{6}\right) c_{4}^{0}-4 c_{0}^{4} c_{4}^{4}, \\
& C_{8}=4\left(c_{2}^{2}+c_{2}^{6}\right)\left(c_{2}^{0}+c_{2}^{8}\right)-4\left(c_{2}^{4}\right)^{2} .
\end{aligned}
$$

The partition function blocks mix under the $S$ modular transformation as $S\left(A_{8}\right)=\frac{1}{2} A_{8}+\frac{1}{2} B_{8}+C_{8}, \quad S\left(B_{8}\right)$ $=\frac{1}{2} A_{8}+\frac{1}{2} B_{8}-C_{8}$, and $S\left(C_{8}\right)=\frac{1}{2} A_{8}-\frac{1}{2} B_{8}$. Equation (9) implies that $A_{8} \sim q^{0}(1+\cdots), B_{8} \sim q^{1 / 2}(1+\cdots)$, and $C_{8} \sim q^{3 / 4}(1+\cdots)$. The massless states only contribute to the terms $2 c_{0}^{0} c_{0}^{2}-2\left(c_{4}^{4}\right)^{2}$ in $A_{8}$. Again, the $q^{0}$ term in $2 c_{0}^{0} c_{0}^{2}$ can be identified with the massless vector state of Eq. (3). The term $2\left(c_{4}^{4}\right)^{2}$ must be interpreted as space-time fermions.

The massless fermion states of the fractional superstrings are described in a manner closely analogous to those of the $K=2$ superstring. They appear in the above partition functions in terms of the form $\left(c_{K}^{K / 2} / 2\right)^{D-2}$. The spin field $\sigma_{K / 2}$ is the lowest dimension parafermion field in $c_{K}^{K / 2}$. We write the fermion ground state as

$$
|\phi\rangle=\prod_{\mu=1}^{D} \sigma_{K / 2}^{\mu}|p\rangle,
$$

in analogy to the Ramond ground state of the superstring. The energy operator $\epsilon^{\mu}$ can have integer moding when acting on $\sigma_{K / 2}^{\mu}$. From the parafermion theory [4], one can show that $\epsilon_{0}^{\mu} \epsilon_{0}^{\mu}=1$ when acting on $\sigma_{K}^{\mu}$. With appropriate Klein factors $\epsilon_{0}^{\mu}$ satisfies the Clifford algebra, $\left\{\epsilon_{0}^{\mu}, \epsilon_{0}^{v}\right\}=-2 g^{\mu v}$, when acting on $|\phi\rangle$, where $g^{\mu v}$ is the Minkowski metric. Thus the $\epsilon_{0}^{\mu}$ can be identified with Dirac gamma matrices and $|\phi\rangle$ has the degeneracy and Lorentz properties of a spinor. $|\phi\rangle$ is massless if we choose the intercept zero for this sector: $L_{0}|\phi\rangle=p^{2}|\phi\rangle$ $=0$. The only nontrivial physical state condition on $|\phi\rangle$ is given by the $G^{K}$ zero mode $G_{0}^{K}=\sum_{n}\left(\alpha_{-n}^{\mu}+c_{n}^{\prime} \epsilon_{-}^{\mu}\right) \epsilon_{n, \mu}$. We find for the physical state condition $0=G_{0}^{K}|\phi\rangle$ $=p \cdot \epsilon_{0}|\phi\rangle=p|\phi\rangle$, giving the massless Dirac equation. With a Weyl and/or Majorana projection, the number of physical spinor degrees of freedom is 8,4 , and 2 in 10,6 , and 4 dimensions, respectively, in agreement with the $Z_{(K, K)}$ partition functions.

Note that the massless spectrum in the closed string partition function $Z_{(K, K)}$ has the correct counting of states for a $D$-dimensional $N=2$ supergravity theory. The existence of massless spin- $\frac{3}{2}$ states suggests that if the theory is to be unitary, it must have space-time supersymmetry. This implies that $Z_{(K, K)}$ vanishes, which in 
turn implies that $A_{K}=B_{K}=C_{K}=0$. We have checked, using known expressions for the string functions [12], that $A_{K}, B_{K}$, and $C_{K}$ are each identically zero out to the first $10^{4}$ terms in their expansions in $q$. It turns out that these identities can indeed be proven [15].

In summary, there exist modular-invariant combinations of the string functions which remove tachyon contributions by projections similar to the Gliozzi-Scherk-Olive projection [13]. The resulting partition functions have only positive and negative integer coefficients, permitting a space-time particle interpretation which is supported by the explicit construction of the massless degrees of freedom. Finally, the partition functions obey an exact cancellation of boson and fermion degrees of freedom at each mass level, indicative of space-time supersymmetry.

The arguments presented in this Letter also suggest other types of new string theories: (1) So far we have built only closed string theories in which the left- and right-moving world-sheet symmetries are matched. However, one can also build new types of heterotic strings in which the left- and right-moving world-sheet symmetries are different [16]. (2) Another possible new string theory has $K=16$ and critical dimension $D=3$ which could describe a theory of space-time anyons. A tachyon-free, supersymmetric $K=16$ partition function will be presented elsewhere [15]. (3) A final possibility is the $K=\infty$ string with $D=2$ where $G^{\infty}$ has unit dimension. It has partition function $Z_{(\infty, \infty)}=$ const. We believe such a string can be constructed by gauging appropriate WZW currents on the string world sheet.

There are clearly many issues that must be addressed in order to show that the fractional superstrings proposed here are consistent theories. Foremost among these are the questions of unitarity, scattering amplitudes, the ghost system, and the local world-sheet symmetry underlying the fractional supersymmetry constraint algebra. The techniques that answer these questions for the $K=2$ superstring depend largely on the fact that the super Virasoro algebra is a local algebra on the world sheet. The $K=4$ fractional supersymmetry chiral algebra is nonlocal; however, there exists a splitting of its current that satisfies an algebra with Abelian (parafermionic) braiding relations, allowing a description of the algebra in terms of generalized commutators of modes [5]. We believe that the $K=8$ and 16 algebras are both nonlocal and non-Abelian [8], and will require new techniques to ap- proach them. The fractional superstrings proposed here thus present conceptual and calculational challenges; they also promise rich and novel world-sheet structures.

It is a pleasure to thank K. Dienes, J. Grochocinski, A. LeClair, and E. Lyman for discussions. This work was supported in part by the National Science Foundation.

[1] See, e.g., M. B. Green, J. H. Schwarz, and E. Witten, Superstring Theory (Cambridge Univ. Press, Cambridge, 1987).

[2] D. Kastor, E. Martinec, and Z. Qiu, Phys. Lett. B 200, 434 (1988).

[3] J. Bagger, D. Nemeschansky, and S. Yankielowicz, Phys. Rev. Lett. 60, 389 (1988); F. Ravanini, Mod. Phys. Lett. A 3, 397 (1988).

[4] A. B. Zamolodchikov and V. A. Fateev, Zh. Eksp. Teor. Fiz. 89, 380 (1985) [Sov. Phys. JETP 62, 215 (1985)].

[5] A. B. Zamolodchikov and V. A. Fateev, Theor. Math. Phys. 71, 451 (1987).

[6] R. Poghossian, Int. J. Mod. Phys. A 6, 2005 (1991).

[7] S. Chung, E. Lyman, and S.-H. H. Tye, Cornell University Report No. CLNS 91/1057 (to be published).

[8] P. C. Argyres, J. Grochocinski, and S.-H. H. Tye, Cornell University Report No. CLNS 91/1059 (to be published).

[9] The tensor product algebra is not equivalent to the single component algebra, and so leads to different critical central charges than those found in P. C. Argyres, A. LeClair, and S.-H. H. Tye, Phys. Lett. B 253, 306 (1991).

[10] L. Brink and H. B. Nielsen, Phys. Lett. 45B, 332 (1973).

[11] A comparison of the critical central charges shows that the fractional superstrings proposed here are unrelated to the theories of F. Ardalan and F. Mansouri, Phys. Rev. Lett. 56, 2456 (1986); Phys. Lett. B 176, 99 (1986); P. H. Frampton and M. R. Ubriaco, Phys. Rev. D 38, 1341 (1988).

[12] V. G. Kac, Adv. Math. 35, 264 (1980); V. G. Kac and D. Peterson, Bull. Am. Math. Soc. 3, 1057 (1980); Adv. Math. 53, 125 (1984); D. Gepner and Z. Qiu, Nucl. Phys. B285, 423 (1987).

[13] F. Gliozzi, J. Scherk, and D. Olive, Nucl. Phys. B122, 253 (1977)

[14] Throughout this Letter, the appropriate powers of $\operatorname{Im} \tau$ in partition functions and of $\tau$ in their modular transformations are suppressed.

[15] P. C. Argyres, K. R. Dienes, and S.-H. H. Tye, Cornell University Report No. CLNS 91/1113 (to be published).

[16] K. R. Dienes and S.-H. H. Tye, Cornell University Report No. CLNS 91/1100 (to be published). 